\title{
ALFABETIZACIÓN AMBIENTAL EN ESTUDIANTES DE INGENIERIIA DE LA UNIVERSIDAD DE SONORA
}

\author{
Environmental literacy in students of Engineering \\ of the university of sonora
}

EPISTEMUS

ISSN: 2007-8196 (electrónico)

ISSN: 2007-4530 (impresa)

I.I.S. Marco Alberto a La Torre Islas 1 DRA. Andrea Zavala Reyna 2

DRA. Juana Alvarado Ibarra ${ }^{3}$

Recibido: 18 de Abril de 2017,

Aceptado: 14 de noviembre de 2017

Autor de Correspondencia:

I.I.S. Marco Alberto a La Torre Islas

Correo: beto180588@hotmail.com

\section{Resumen}

La presente investigación muestra el nivel de alfabetización ambiental de los alumnos de la División de Ingeniería de la Universidad de Sonora en cuanto a las variables: actitud ambiental, conducta ambiental y conocimiento ambiental, derivados de la aplicación de un instrumento de investigación diseñado por el Centro de Educación Ambiental en Wisconsin (WCEE), E.U.A. de ingeniería es bajo (58.79\%); mientras que el análisis por componentes ambientales, indica que el nivel de actitud ambiental fue de $71.76 \%$; en cambio en conducta ambiental y conocimiento ambiental se obtuvieron valores promedio de $47.69 \%$ y $56.92 \%$ respectivamente. Asimismo, se muestra el análisis de los resultados por género.

\section{Abstract}

This research shows the level of environmental literacy of students in the Engineering Division of the University of Sonora in terms of environmental attitudes, environmental behavior and environmental knowledge, results obtained through the application of a data collection instrument designed by the Wisconsin Center for Environmental Education (WCEE), E.U.A. Results demonstrate that the level of environmental literacy in students is low (58.79\%); in so far as environmental analysis components, the level of environmental attitude was 71.76\%; as to the results of environmental behavior and environmental knowledge was obtained a mean value of $47.69 \%$ and $56.92 \%$ respectively. Also, it is shown the results obtained by gender. 


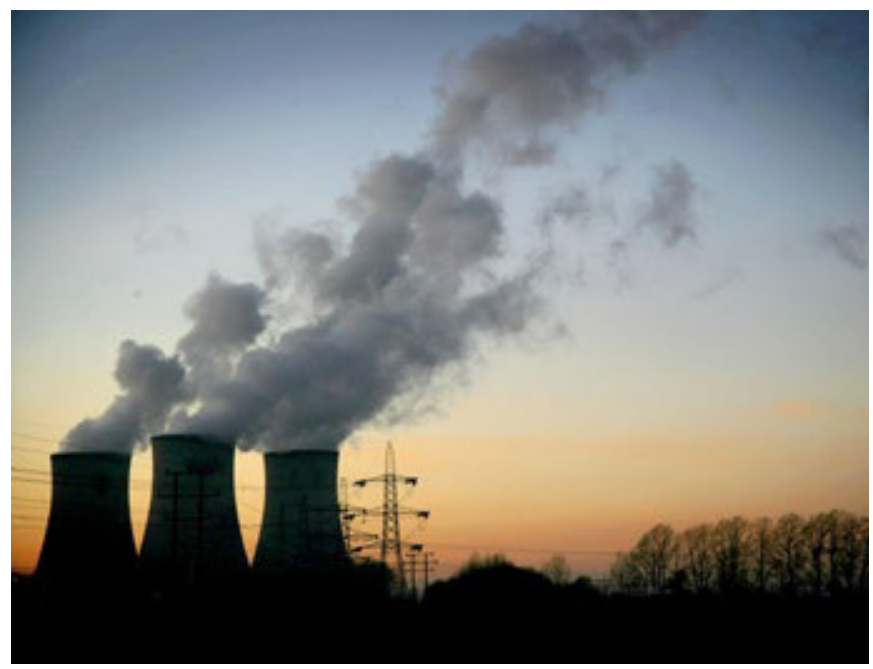

\section{INTRODUCCIÓN}

Actualmente la humanidad se encuentra bajo el llamado "dilema ambiental", el cual, según Owen (1), es resultado de cuatro factores principales: el rápido incremento de la población, la contaminación, el excesivo consumo de recursos y el gradual deterioro de una ética de la Tierra. Este dilema puede ser perfectamente manejable con la ayuda de la postura del desarrollo sustentable si se hacen compatibles las necesidades humanas con las de los ecosistemas (2) y tomando como primicia examinar con especial atención el modo en que la educación puede contribuir para alcanzar el desarrollo sustentable y mejorar las perspectivas de equidad y paz en el mundo (3). En este respecto, la Organización de las Naciones Unidas para la Educación, la Ciencia y la Cultura (UNESCO),sostiene que la educación es esencial para alcanzar el desarrollo sustentable, debido a que ésta es crucial para que los líderes y ciudadanos del mañana desarrollen la habilidad de encontrar soluciones y crear nuevos senderos hacia un futuro mejor, razón por la cual en el año 2002 la Asamblea General de las Naciones Unidas creó el llamado Decenio de las Naciones Unidas sobre la Educación para el Desarrollo Sustentable (4), el cual reconoce la urgente necesidad de integrar los temas y los principios del desarrollo sustentable en la educación y el aprendizaje (5).

Para Bravo (6), la educación superior no está exenta de esta necesidad, por lo que debe contribuir reorientando el estilo de desarrollo hacia grados crecientes de sustentabilidad a través de su trabajo educativo. Como un resultado a esta demanda, gran cantidad de instituciones

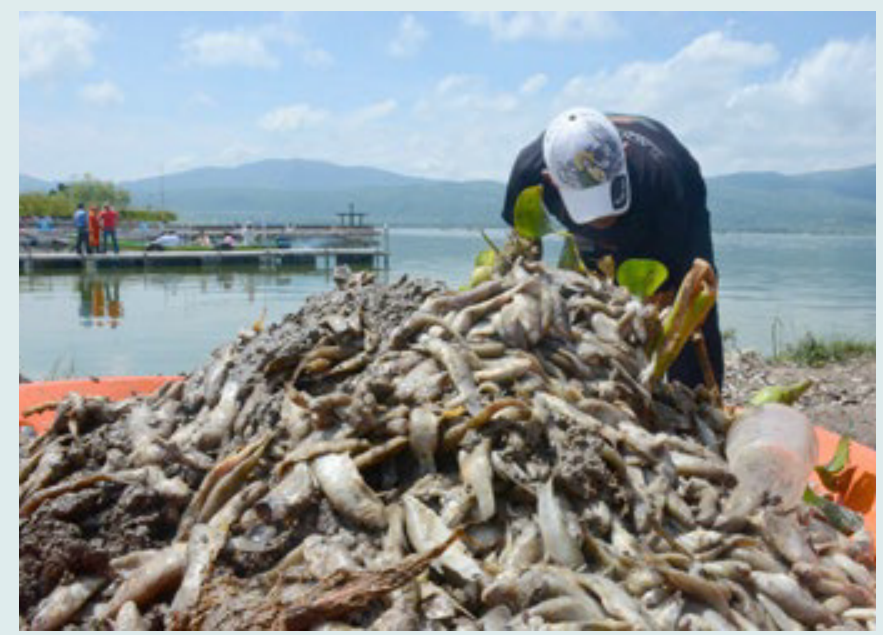

han incorporado la educación ambiental a su currículo, con la idea que el desarrollo de conocimientos y actitudes proambientales permitirán formar ciudadanos preocupados por el impactoquetiene su comportamiento en el ambiente; no obstante, varios estudios han mostrado que no existe una relación directa entre el conocimiento, las actitudes y el comportamiento pro-ambiental o sustentable (7). En este contexto, Isaac-Márquez y colaboradores argumentan que para elevar el nivel de cultura ambiental de los jóvenes es necesario, en primera instancia, el reconocimiento de la educación ambiental como una prioridad por parte de todos los actores del sector educativo (8) y otorgarle más recursos, más infraestructura y que sea materia obligatoria en todos los niveles del sistema educativo formal, como eje transversal del conocimiento y en la forma de cursos curriculares específicos. Adicionalmente, la educación ambiental requiere de situaciones pedagógicas que vayan más allá de la simple transmisión - información, que comprendan las condiciones de sujeto pensante, afectivo, moral y estético, mediante las cuales se logre el desarrollo de un ser integral en la búsqueda de soluciones a los problemas ambientales (9), para esto se requiere que los alumnos cuenten con un grado de alfabetización ambiental; es decir, que cuenten con un conjunto de mecanismos cognitivos y afectivos en pro del cuidado del ambiente, entre los cuales destacan el definir, situar y reconocer los problemas y sus consecuencias, admitir que nos afectan, valorar nuestro papel como importante, desarrollar el deseo, sentir la necesidad de tomar parte de la solución y elegir las mejores estrategias con los recursos más idóneos (9). Además de actitud ambiental, la cual según Zimmerman se refiere al proceso 
psico-socio-ambiental de evaluación del individuo frente al ambiente externo, con fines adaptativos y para la toma de decisiones en sus locomociones diarias (10); no obstante, no basta contar con actitud ambiental, sino que además es importante demostrar una conducta ambiental que se refiere a la acción que realiza una persona, ya sea de forma individual o en un escenario colectivo, a favor de la conservación de los recursos naturales y dirigida a obtener una mejor calidad del medio ambiente (11). Por último y de acuerdo a Febles, es necesario contar con conocimiento ambiental referido a la obtención, análisis y sistematización por parte del individuo de la información proveniente de su entorno, social por naturaleza. Éste constituye un paso importante para su comprensión a través de acciones concretas, que a su vez influyen en el desarrollo de estos conocimientos (12). Ante estos requerimientos de formación ambiental en los alumnos, es importante conocer el nivel de alfabetización ambiental de los estudiantes de la Universidad de Sonora, así como su nivel de conducta, actitud y conocimiento ambiental; a fin de conocer el impacto que ha tenido en ellos las acciones llevadas a cabo por la institución en los últimos años, sobre todo en la División de Ingeniería, donde desde el 2004 se imparte la materia sustentabilidad en las ingenierías a todos los alumnos.

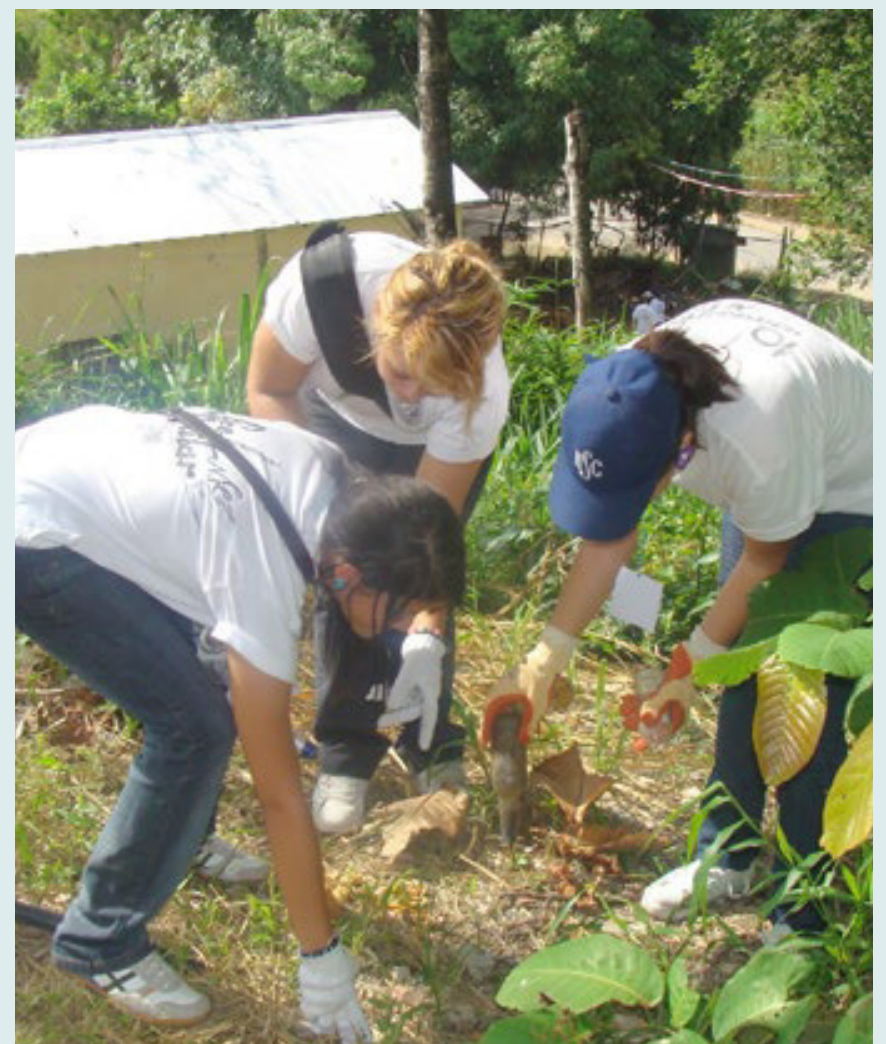

\section{Metodología}

La presente investigación posee un enfoque cuantitativo, ya que se generó información numérica mediante la medición de los niveles de actitudes, conductas y conocimientos hacia el medio ambiente, así como el nivel de alfabetización ambiental correspondiente en los estudiantes del sexto y octavo semestre de la División de Ingeniería del campus centro de la Universidad de Sonora.

Para la selección y tamaño de muestra se eligieron los alumnos inscritos en el semestre 2013-1 en los programas Ingeniería Civil, Ingeniería en Sistemas de Información, Ingeniería Industrial y de Sistemas, Ingeniería Mecatrónica, Ingeniería en Minas e Ingeniería Química.

Con base en los datos proporcionados por la Dirección de Servicios Escolares de la Universidad de Sonora, en el ciclo 2013-1 se encontraban inscritos 1270 estudiantes en la División de Ingeniería. Para la obtención del tamaño de muestra del estudio se utilizaron las ecuaciones 1 y 2 de muestreo aleatorio simple con población finita, con un nivel de confianza del $95 \%$.

\section{Ecuación 1:}

$$
\begin{aligned}
& n=\frac{N p q}{(N-1) D+p q}=\underline{1270(0.5)(0.5)}=305 \text { Encuestas } \\
& (1270-1)(0.000625)+(0.5)(0.5)
\end{aligned}
$$

\section{Ecuación 2:}

$D=\frac{B^{2}}{4}=(0.05)^{2} / 4=0.000625$

Donde:

Con $95 \%$ de confianza $p=0.5$ (proporción)

$q=0.5$

$B=(B$ es el error de $5 \%)$

$B^{2}=0.05^{2}$

$N=1270$ (Total de alumnos activos en el semestre 2013-1)

Posteriormente se aplicó muestreo estratificado por asignación proporcional, para tener una mayor confianza en que se incluyeran todas las ingenieras de interés en el estudio.

La ecuación 3 fue utilizada para el muestreo estratificado por asignación proporcional y los resultados se muestran en la tabla 1.

\section{Ecuación 3:}

$$
n_{i}=n\left(N_{i} / N\right)
$$

Donde:

$n=$ Total de estudiantes de ingeniería " $x$ " activos en el ciclo 2013-1.

$n_{i}=$ Número de estudiantes de ingeniería " $\mathrm{x}$ " $\mathrm{a}$ aplicarse el instrumento.

$N=$ Total de estudiantes de la división de ingeniería activos en el ciclo 2013-1.

$N_{i}=$ Tamaño de muestra aleatorio simple obtenido de $N$. 
Tabla 1.Tamaños de muestra para el estudio de alfabetización ambiental.

\begin{tabular}{|l|c|c|}
\hline \multicolumn{1}{|c|}{ Programa Académico } & $\begin{array}{c}\text { Estudiantes } \\
\text { que ingresaron } \\
\text { en 2009-2 y } \\
\text { 2010-2 avtivos al } \\
\text { 2013-1 }\end{array}$ & $\begin{array}{c}\text { Tamaño } \\
\text { de } \\
\text { muestra }\end{array}$ \\
\hline Ing. Civil & 364 & 88 \\
\hline Ing.en Sistemas de Información & 103 & 25 \\
\hline In. Industrial y de Sistemas & 346 & 83 \\
\hline Ing. Mecatrónica & 177 & 43 \\
\hline Ing. Minero & 149 & 36 \\
\hline Ing. Química & 127 & 30 \\
\hline Total de estudiantes & 1,270 & 305 \\
\hline
\end{tabular}

En la primera parte de la Encuesta de Nivel de Alfabetización Ambiental diseñada por el Centro de Educación Ambiental en Wisconsin (WCEE) de Estados Unidos Americanos (EUA), se encuentra un apartado que permite recolectar datos generales del encuestado (nivel académico, edad y género) y posteriormente se divide en tres secciones ( $A, B$ y $C$ ) referentes a actitudes ambientales, comportamientos ambientales y conocimientos ecológicos básicos.

Para responder las oraciones de la sección A de actitud ambiental, se emplea la escala de Likert: 1) Fuertemente de acuerdo, 2) De acuerdo, 3) Sin opinión, 4) Desacuerdo y 5) Fuertemente en desacuerdo.

La sección $B$ de comportamiento ambiental, también se utilizó la escala de Likert con las siguientes opciones: 1) Siempre, 2) Casi siempre, 3) Algunas veces, 4) Casi nunca y 5) Nunca.
La sección C, de conocimientos, fue de opción múltiple con 4 posibles respuestas de las cuales solo una es la correcta.

Cada sección del cuestionario fue analizada por separado para obtener un índice de actitudes, conductas y conocimientos. Para tal efecto, se codificaron los valores de la escala de Likert, asignando un valor de 0 a la opción menos deseable y una puntuación de 4 a la más deseable desde el punto de vista ambiental. En el caso de la sección de conocimientos, se asignó un valor de 4 a la respuesta correcta y un valor de cero a las incorrectas. De esta manera el valor mínimo posible de cada sección es de cero y el máximo de 60.

Las puntuaciones obtenidas en cada sección fueron sumadas para obtener el nivel de alfabetización ambiental. Dichas puntuaciones fueron evaluadas utilizando una escala del calificación base 100, misma que se describe a continuación: de 90 a 100 es excelente, 80 a 89, es muy aceptable, de 70 a 79 es aceptable, de 60 a 69 es inaceptable y menos de 60 corresponde a un nivel bajo.

Para manipulación y análisis de datos se utilizó una hoja de cálculo de Microsoft Office Excel y el paquete estadístico SPSS versión 17.0.

\section{RESULTADOS}

\section{Nivel de actitud ambiental}

En la figura 1 se muestra el comportamiento estadístico de los resultados de los estudiantes encuestados en cuánto a la actitud ambiental. La media obtenida en el alumnado de ingeniería fue de 43.06 (71.76\%), y la desviación estándar resultó de 9.22 , lo cual nos indica que los alumnos de Ingeniería de la UNISON tienen un nivel de actitud ambiental aceptable.

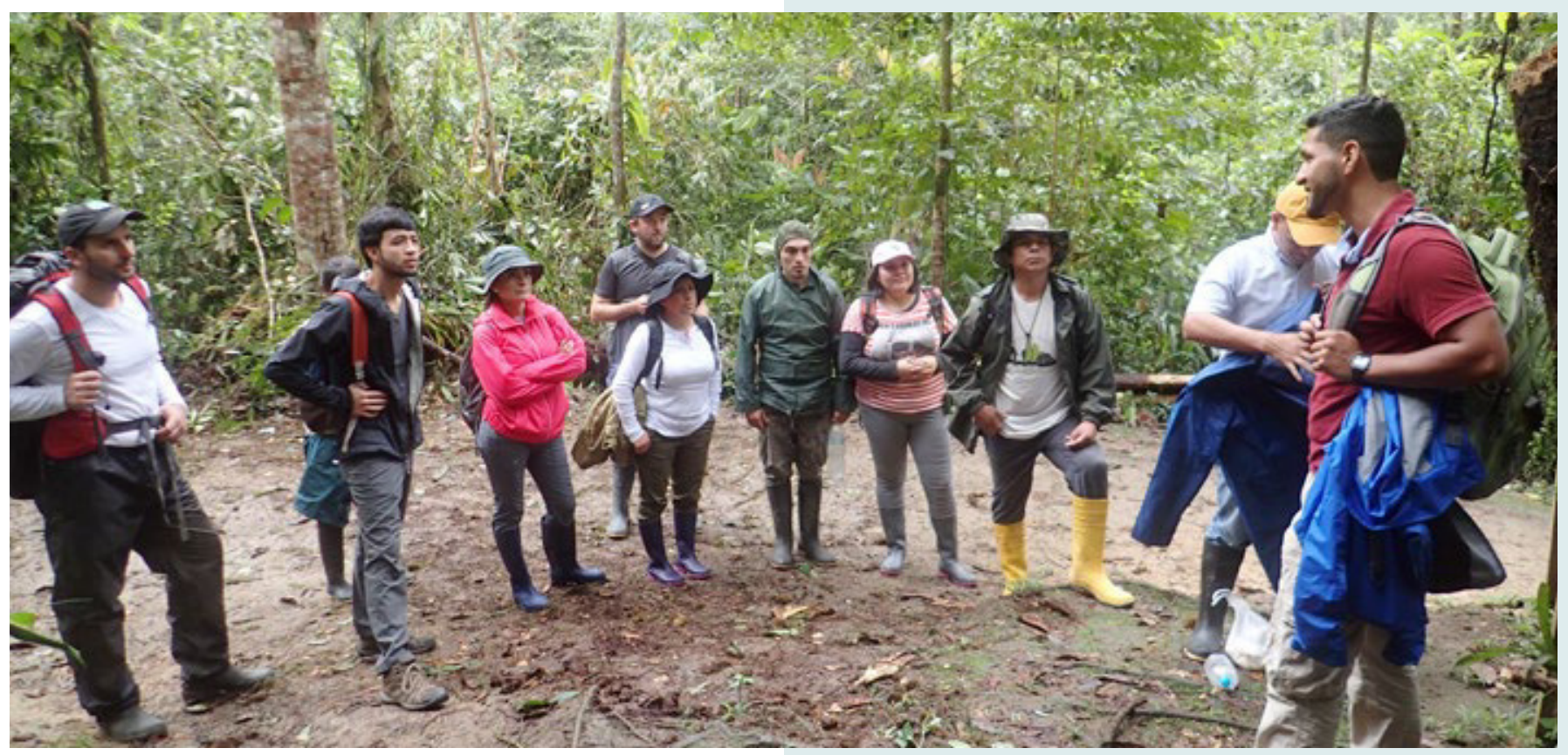




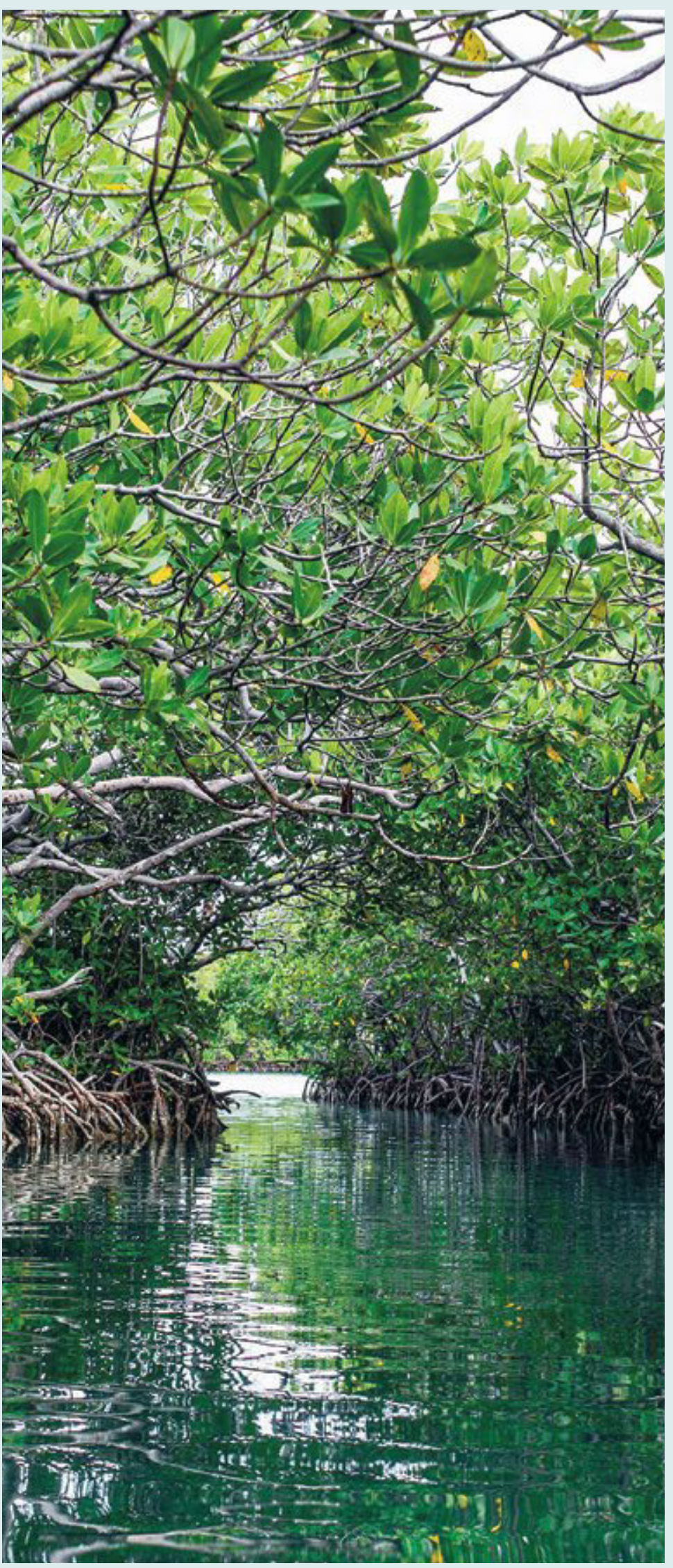

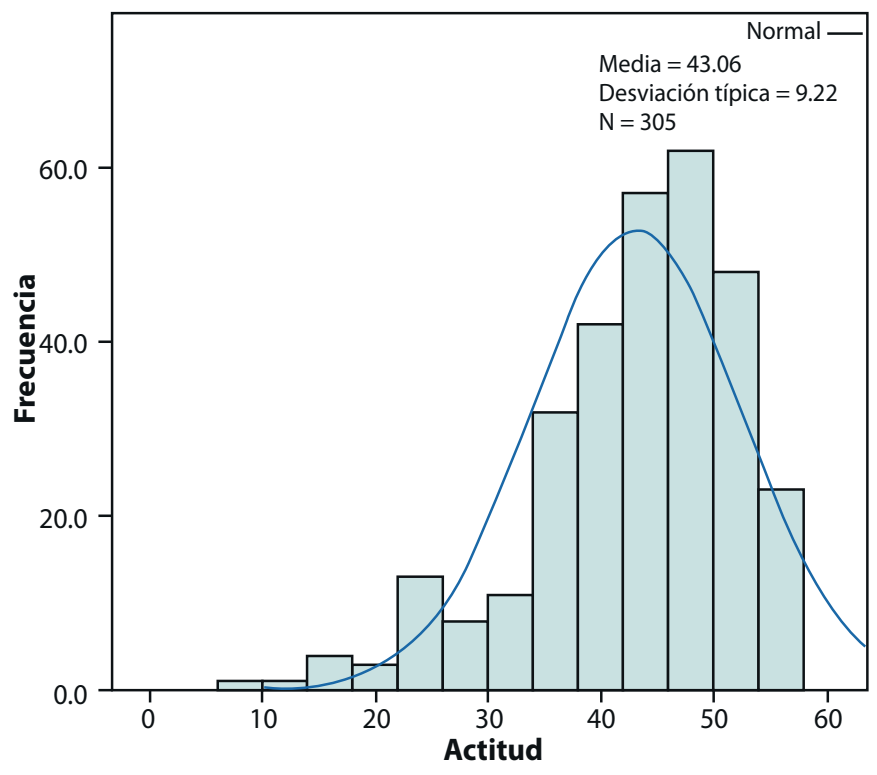

Figura 1. Nivel de actitud ambiental en estudiantes de ingeniería de la UNISON.

\section{Nivel de conducta ambiental}

En la figura 2 se presentan los resultados correspondientes a la determinación del nivel de conducta ambiental. La media obtenida en el estudio fue de 28.62 (47.69\%), con una desviación estándar resultante de 12.33, lo cual indica un nivel de conducta ambiental bajo.

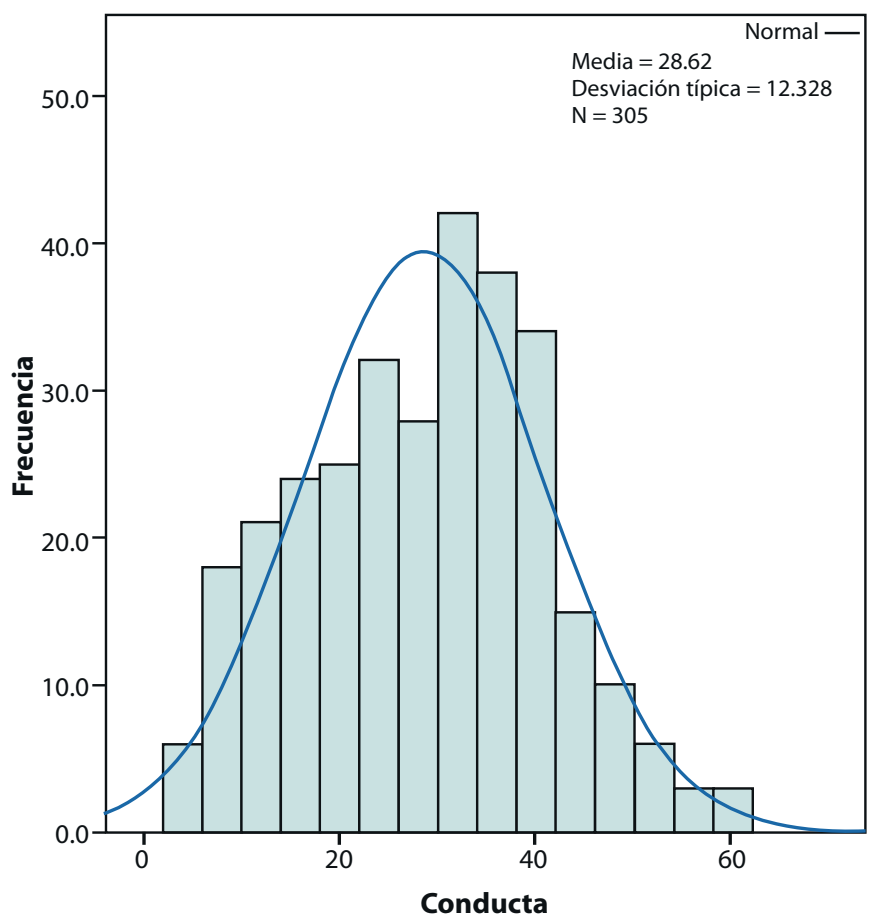

Figura 2. Nivel de conducta ambiental en estudiantes de ingeniería de la UNISON. 


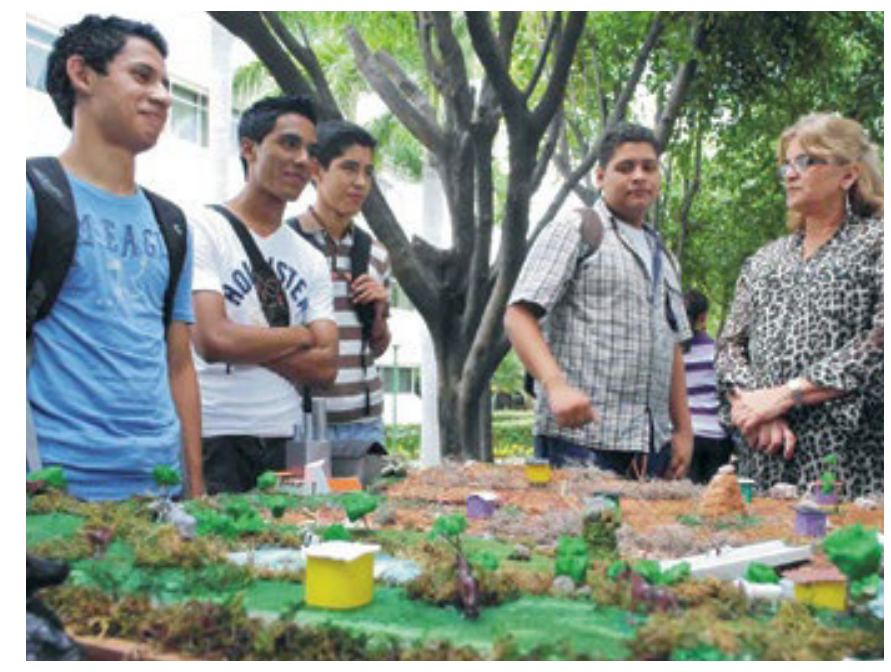

Nivel de conocimiento ambiental

La figura 3 muestra los resultados referentes al nivel de conocimiento ambiental. La media resultante en el estudio fue de 34.15 (56.92\%), con una desviación estándar de 10.04 , lo cual indica un nivel de conocimiento ambiental bajo.

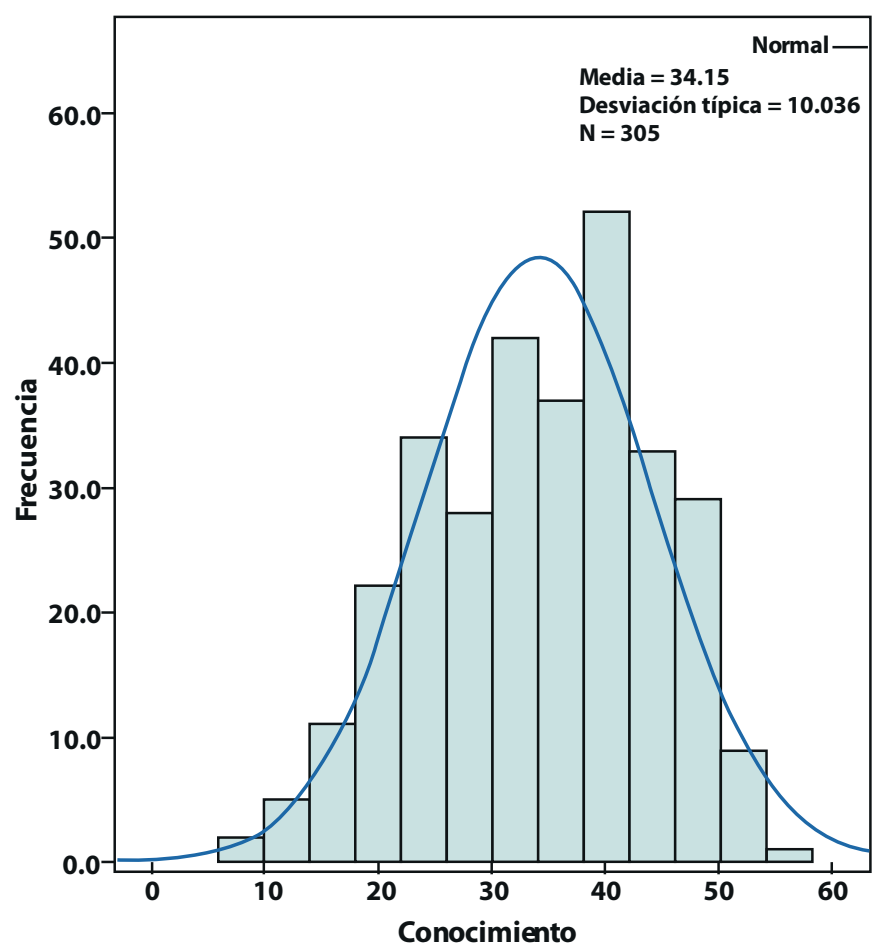

Figura 3. Nivel de conocimiento ambiental en estudiantes de ingeniería de la UNISON.

Nivel de alfabetización ambiental (integración de actitudes, conductas y conocimientos ambientales)

De manera conjunta, el nivel de Alfabetización Ambiental alcanzado por los estudiantes encuestados fue de 105.82 (58.79\%), con una desviación estándar de 20.94 . Lo anterior se presenta en la figura 4, lo cual indica que la Alfabetización Ambiental del alumnado se encuentra en el nivel bajo.

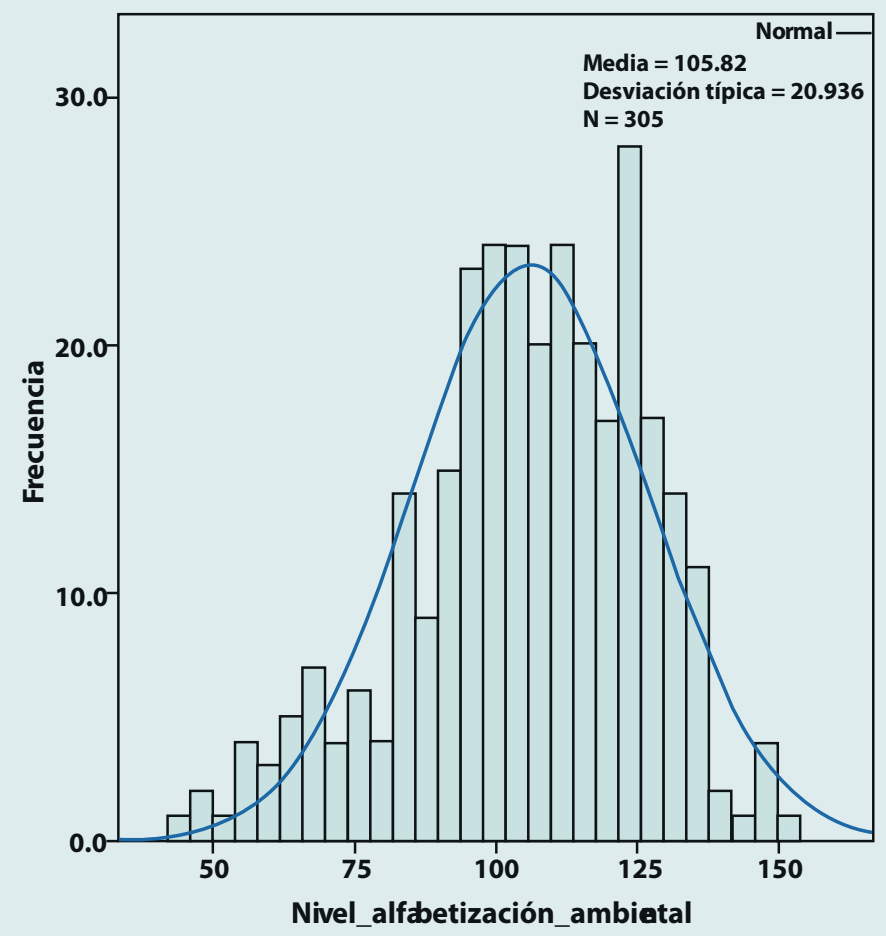

Figura 4. Nivel de alfabetización ambiental en estudiantes de ingeniería de la UNISON.

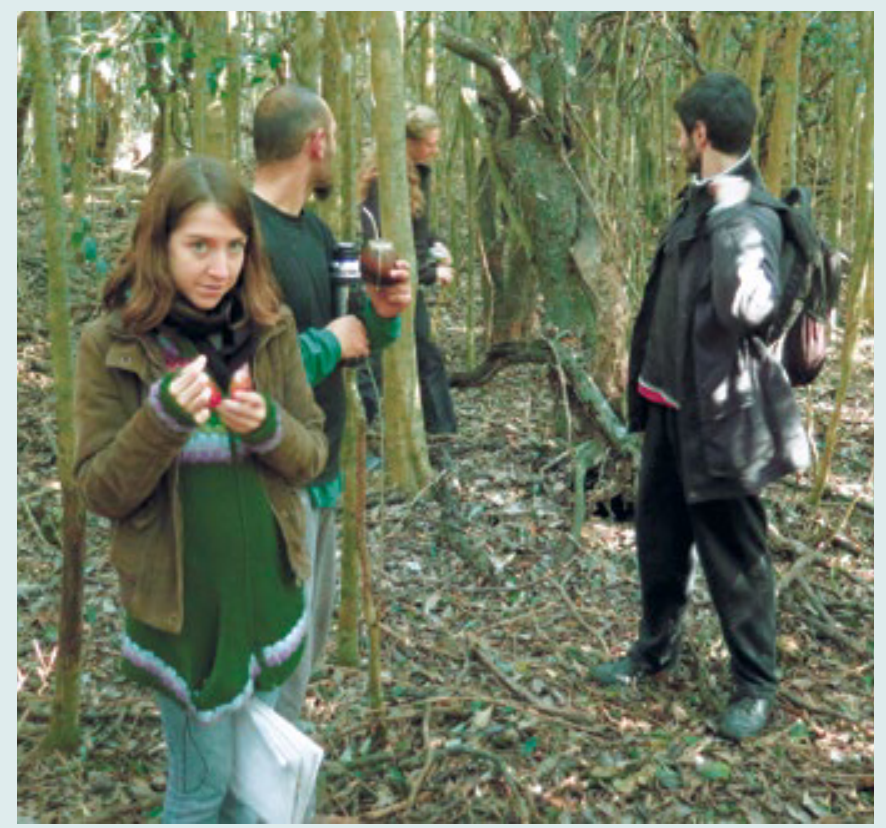




\section{DISCUSIÓN}

El instrumento de alfabetización ambiental empleado en la presente investigación ha sido utilizado anteriormente por diversos investigadores, tal es el caso de Courtney (13) con los estudiantes de la Universidad de Florida, así como Montaño y colaboradores (2012) en los estudiantes de ingeniería en ciencias ambientales del Instituto Tecnológico de Sonora (ITSON) (14). La tabla 4, muestra una comparativa de los resultados obtenidos entre esas instituciones y la Universidad de Sonora, donde destaca el hecho de que los alumnos de ingeniería no obtuvieron un nivel inaceptable en ninguna de las variables analizadas, a pesar de no tratarse de programas netamente ambientales, como en el caso del ITSON.

Tabla 4. Comparación de estudios respecto a las variables Actitud, Conducta, Conocimiento y Alfabetización ambiental.

\begin{tabular}{|c|c|c|c|c|}
\hline & $\begin{array}{l}\text { Nivel de } \\
\text { Actitud }\end{array}$ & $\begin{array}{l}\text { Nivel de } \\
\text { Conducta }\end{array}$ & $\begin{array}{c}\text { Nivel de } \\
\text { Conocimiento }\end{array}$ & $\begin{array}{c}\text { Nivel de } \\
\text { alfabetización } \\
\text { ambiental }\end{array}$ \\
\hline \multirow{2}{*}{$\begin{array}{l}\text { Estudiantes de } \\
\text { Universidad } \\
\text { de Florida }\end{array}$} & $70.5 \%$ & $39.0 \%$ & $65.5 \%$ & $58.3 \%$ \\
\hline & Aceptable & Nivel Bajo & Nivel Aceptable & Nivel Bajo \\
\hline \multirow[b]{2}{*}{$\begin{array}{l}\text { Estudiantes } \\
\text { ICA de ITSON }\end{array}$} & $77.83 \%$ & $68.51 \%$ & $63.81 \%$ & $70.08 \%$ \\
\hline & Aceptable & $\begin{array}{c}\text { Nivel } \\
\text { Inaceptable }\end{array}$ & $\begin{array}{c}\text { Nivel } \\
\text { Inaceptable }\end{array}$ & $\begin{array}{c}\text { Nivel } \\
\text { Aceptable }\end{array}$ \\
\hline \multirow{2}{*}{$\begin{array}{l}\text { Estudiantes } \\
\text { Ingeniería } \\
\text { UNISON }\end{array}$} & $71.76 \%$ & $47.69 \%$ & $56.92 \%$ & $58.79 \%$ \\
\hline & Aceptable & Nivel Bajo & Nivel Bajo & Nivel Bajo \\
\hline
\end{tabular}

Por último, en la tabla 5 se presenta una comparación de los 3 estudios mencionados con respecto al género de los alumnos encuestados. Los resultados de Courtney (13) en la Universidad de Florida son muy similares a los del alumnado de ingeniería de la Universidad de Sonora; mientras que los estudiantes, tanto hombres como mujeres de ingeniería en ciencias ambientales de ITSON (14) resultaron con un nivel de alfabetización ambiental superior.

Tabla 5. Comparación de los estudios respecto a la variable género.

\begin{tabular}{|l|c|c|c|c|}
\hline & Mujeres & $\begin{array}{c}\text { Nivel de } \\
\text { alfabetización }\end{array}$ & Hombres & $\begin{array}{c}\text { Nivel de } \\
\text { alfabetización }\end{array}$ \\
\hline $\begin{array}{l}\text { Estudiantes de } \\
\text { Universidad de } \\
\text { Florida }\end{array}$ & $58.60 \%$ & Bajo & $58.12 \%$ & Bajo \\
\hline $\begin{array}{l}\text { Estudiantes ICA } \\
\text { de ITSON }\end{array}$ & $70.83 \%$ & Aceptable & $69.41 \%$ & Inaceptable \\
\hline $\begin{array}{l}\text { Estudiantes } \\
\text { Ingeniería } \\
\text { UNISON }\end{array}$ & $58.86 \%$ & Bajo & $58.76 \%$ & Bajo \\
\hline
\end{tabular}

\section{CONCLUSIONES}

La aplicación del instrumento de investigación diseñado por el Centro de Educación Ambiental en Wisconsin (WCEE), EUA, ha servido para conocer el nivel de alfabetización ambiental en los estudiantes de sexto y octavo semestre de los programas de ingeniería de la Universidad de Sonora es bajo (58.79\%). El análisis por componentes nos permitió conocer que los alumnos

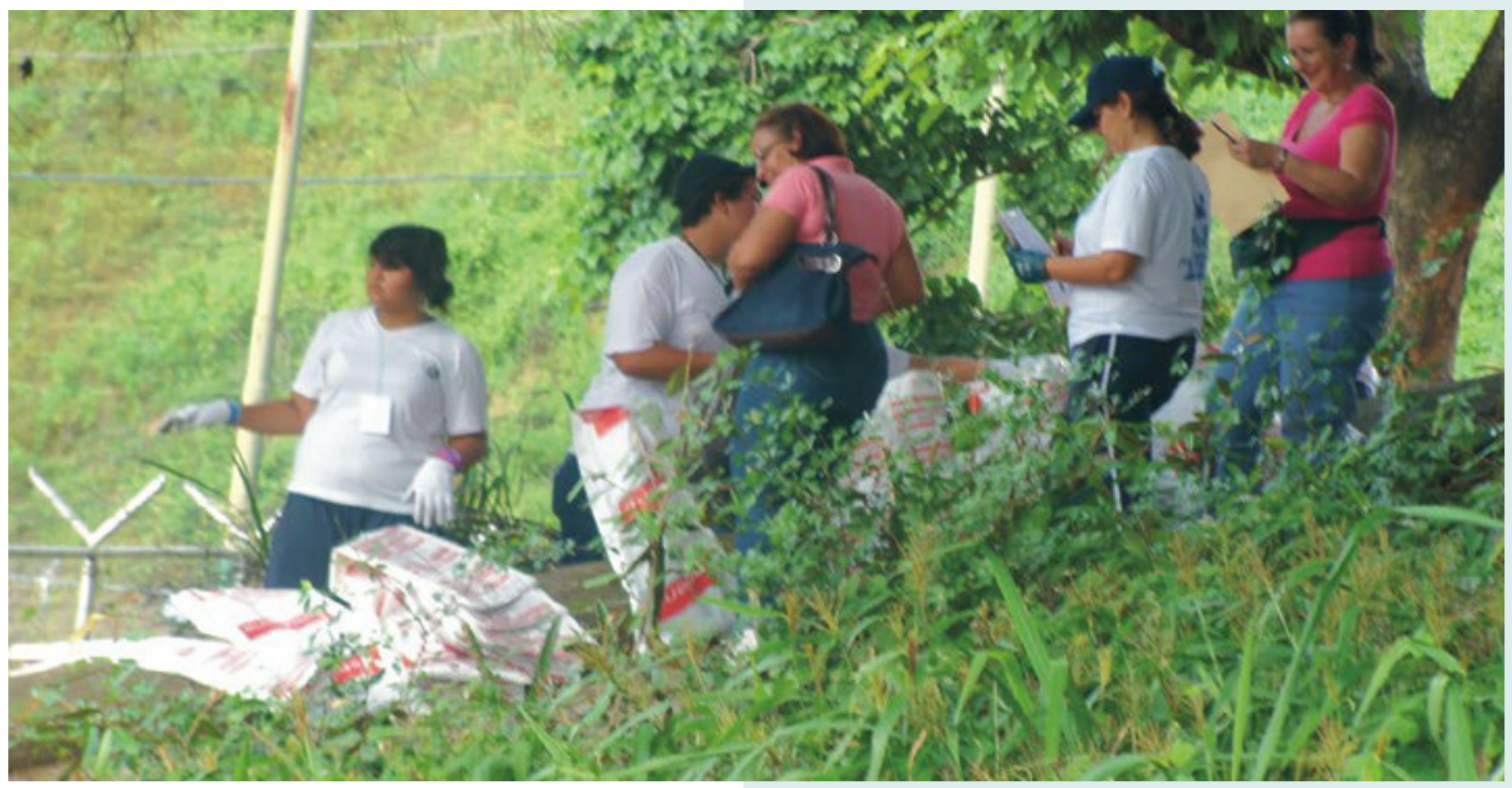


cuentan con un nivel de actitud aceptable (71.76\%), pero obtuvieron niveles bajo para las variables de conducta y conocimientos ambientales, como fueron $47.69 \%$ y $56.92 \%$ respectivamente.

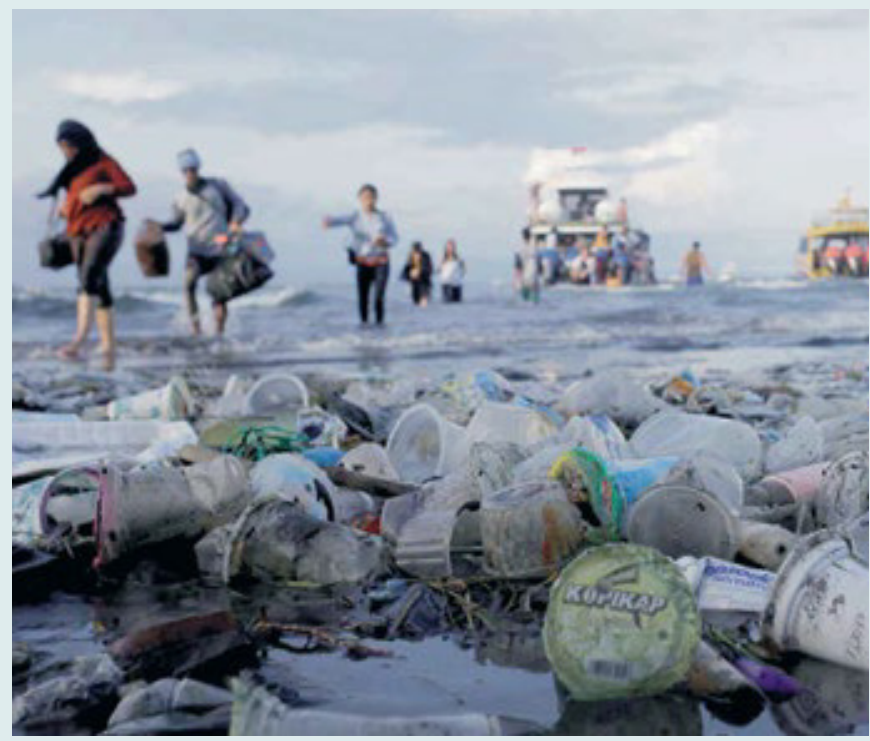

Por lo anterior, se concluye que la asignatura de "Sustentabilidad en las ingenierías" y otras relacionadas han contribuido de manera positiva en la actitud de los estudiantes de ingeniería hacia el cuidado del medio ambiente, sobre todo porque, a diferencia del estudio realizado en el ITSON, los alumnos no son especialistas en el área ambiental. Sin embargo, a pesar de que las asignaturas relacionadas han contribuido a la formación de los estudiantes en esta área, la impartición de asignaturas no es suficiente y se requiere de una formación holística en los estudiantes en dónde se debe ofrecer una formación académica de calidad que incluya tópicos ambientales, también se les deben inculcar valores a través de su participación en proyectos dentro y fuera del campus, que les muestren la realidad que vivimos en términos de la situación ambiental, a fin de que sean personas y profesionistas respetuosos de su entorno que coadyuven a la sociedad en sus transición a estilos de vida más sustentables.

\section{BIBLIOGRAFÍA}

1) Owen, O. (2000). Recursos naturales. (e-book) Nueva York: The Macmillan Company. Disponible en: Google Books http://books.google.es/books?id=0Z_KmG0yOvEC\&pg= PA1\&dq =dilema+ambiental\&hl=es\&sa $=X \& e i=t$ XvoUZX BJPQ8wTdu4FI\&ved=0CDsQ6AEwAg (Accesado 23 de Junio del 2013)

2) Corral, V. (2010). Psicología de la sustentabilidad. Hermosillo: Universidad de Sonora. Editorial Trillas, ISBN

3) Macedo, B., y Salgado, C. (2007). Educación ambiental y educación para el desarrollo sostenible en América Latina, Forum de sostenibilidad (online) Disponible en: http://www. ehu.es/cdsea/web/revista/numero_1/01_03macedo.pdf (Accesado 3 de Julio del 2013)
4) UNGS (2005). United Nations Decade of Education for Sustainable Development. UN, A/RES/59/237

5) UNESCO (2005). International implementation scheme for the UN decade of education for sustainable development (2005-2014). UNESCO International Science, Technology \& Environmental Education Newsletter, 30, 1-1.

6) Bravo, M.M.T. (2012). La UNAM y sus procesos de ambientalización curricular. Revista Mexicana de Investigación Educativa, 17, 1119-1146.

7) Escobar, M.S. (2012). Comportamiento sustentable y educación ambiental: una visión desde las prácticas culturales. Revista Latinoamericana de Psicología, 44, 181-196.

8) Vega, M. P. y Álvarez, S.P. (2005). Planteamiento de un marco teórico de la educación ambiental para un desarrollo sostenible. Revista electrónica de enseñanza de las ciencias, 4 (1).

9) Isaac-Márquez, R., Salavarría, O., Eastmond, A., Ayala, M., Arteaga, M., Isaac-Márquez, A., Sandoval, V., Manzanero, A. (2011). Cultura ambiental en estudiantes de bachillerato. Estudio de caso de la educación ambiental en el nivel medio superior de Campeche. Revista Electrónica de Investigación Educativa, 13(2), 83-98.

10) Serna, R.A. (2007). El derecho a un ambiente sano y la pedagogía ambiental. Revista El Ágora USB, 7, 345-359.

11) Zimmermann, M. (2005). Ecopedagogía: el planeta en emergencia. Bogotá: Ecoe ediciones.

12) Puertas, V. S. y Aguilar L. M. C. (2008). Psicología ambiental. Departamento de Psicología. Universidad de Jaén. Recuperado de http://www4.ujaen.es/ spuertas/Private/ Tema\%209.pdf

13) Febles, M. (2001). Bases para una Psicología Ambiental en Cuba. Facultad de Psicología. Universidad de La Habana.

14) Courtney, N. (2002). An analysis of the correlations between the Attitude, Behavior, and knowledge, components of Environmental Literacy in undergraduate university students. Recuperado de http://ufdc.ufl.edu/UF00100691/00001

15) Montaño S., Cervantes B., Gutiérrez C., Zarate O. (2012). Alfabetización Ambiental en estudiantes de ingeniería en ciencias ambientales del Instituto Tecnológico de Sonora, Revista Desarrollo Local Sostenible. Obtenido en Noviembre 5, 2013, de http://www.eumed.net/rev/delos/14/sbco.pdf

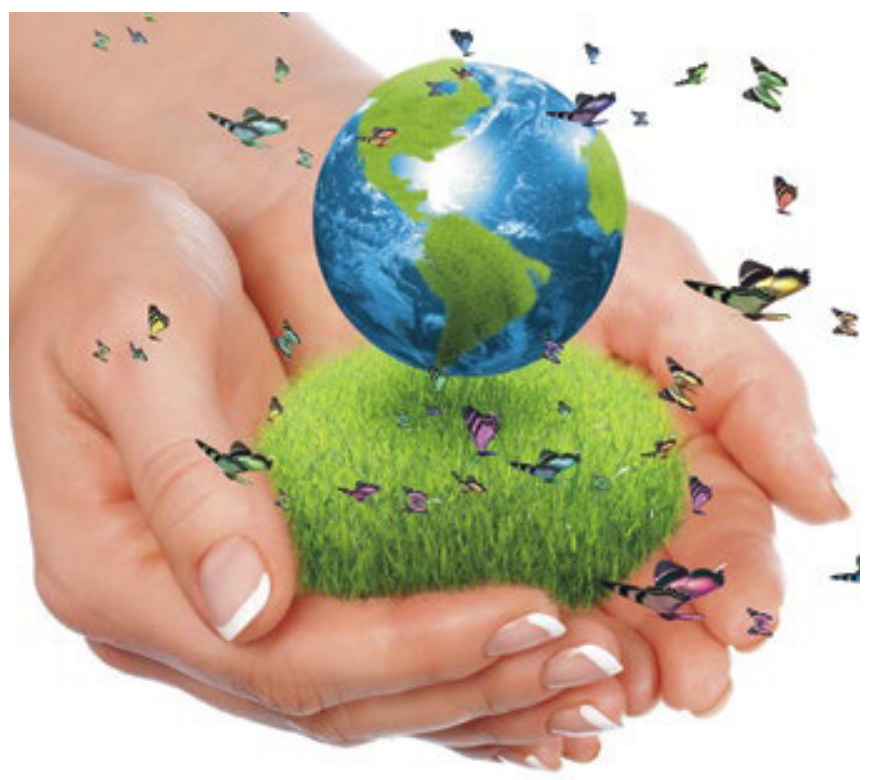

